International Journal of Fatigue

Volume 29, Issue 7, July 2007, Pages 1359-1373

doi:10.1016/i.jifatigue.2006.10.021

(c) 2006 Elsevier Ltd All rights reserved
Archimer, archive institutionnelle de l'Ifremer http://www.ifremer.fr/docelec/

\title{
Life-time reliability based assessment of structures submitted to thermal fatigue
}

\author{
Z. Guédéa, ${ }^{\star}$, B. Sudret ${ }^{b}$ and M. Lemaire ${ }^{c}$ \\ anstitut Français de Recherches pour l'Exploitation de la Mer (IFREMER), Centre de Brest, BP 70, F-29280 \\ Plouzane, France \\ ${ }^{b}$ EDF R\&D, Site des Renardières, 77818 Moret-sur-Loing Cedex, France \\ ${ }^{\mathrm{C} I n s t i t u t}$ Français de Mécanique Avancée - LaMI - Campus des Cézeaux - 63175 Aubière Cedex, France \\ *: Corresponding author : Zakoua.Guede@ifremer.fr
}

\begin{abstract}
A probabilistic approach of the current thermal fatigue design of nuclear components is set up. It aims at incorporating all kinds of uncertainties that affect the thermal fatigue behaviour. This approach is based on the theory of structural reliability. Two dual approaches of reliability analysis for the thermal fatigue are defined, respectively, in the time domain and in the frequency domain. Beside the probability of failure calculation, the sensitivity of the reliability index to each random variable is estimated. The proposed method is applied to a pipe submitted to thermal loading due to water flow. Both high cycles fatigue (HCF) and low cycles fatigue (LCF) regimes are investigated. The results show that it is possible to perform a complete reliability analysis to compute the failure probability. It is observed that the scatter of fatigue data and the heat transfer coefficient are the most important variables in thermal fatigue reliability.
\end{abstract}

Keywords: Life-time; Thermal fatigue; Structural reliability; Spectral methods 


\section{INTRODUCTION}

The behaviour of structures submitted to thermal fatigue is affected by numerous uncertainties due to the statistical variability of thermal loading, the large scatter observed in fatigue test data, the random nature of the thermo-mechanical parameters ( $e$. g. the material properties, the dimensional variations, the surface smoothness, etc.) and the lack of knowledge on the physical phenomenon. The phenomenon of thermal fatigue concerns in particular the nuclear industry, where structural components, such as pipes or turbine blades, are subject to thermal loading. Two nuclear design codes are usually used to assess fatigue life time: the American ASME code, in section III [1], and the French RCC-M code [2]. Both of them take into account the uncertainties in the design process by applying empirical safety margin factors in order to make the design conservative. They do not allow for a quantification of the associated risk yet. Noting that a safe design in random context concerns structural reliability analysis, it is therefore interesting to introduce a global probabilistic framework as an alternative to the current thermal fatigue design code. This requires:

- To set up realistic probability density functions of the random variables involved in the thermal fatigue model,

- To incorporate these random variables in the design procedure to compute the random life-time of the structure under consideration.

In this way, it is possible to estimate the risk associated to the retained design and the effects of each uncertainty onto the reliability of the structure.

The aim of the present paper is to propose a probabilistic approach of the current thermal fatigue design of nuclear components including all kinds of uncertainties that affect the thermal fatigue behaviour. 
First of all, we recall the main steps of the actual thermal fatigue design. Then the principal sources of uncertainties arising in this design are identified and a choice for their probabilistic modelling is suggested. Two formulations of the resulting random damage are derived. Then, the method for thermal fatigue reliability analysis is presented. Finally, an application example of a pipe submitted to variable thermal loading is given.

\section{CLASSICAL THERMAL FATIGUE DESIGN}

The current thermal fatigue design of nuclear components contains the following main items as shown in Figure 1 (e. g. RCC-M [2]):

- The definition of a deterministic thermal loading history $\theta(t)$ which describes the loading scenario. It is given by a periodic temperature sequence, which may be either representative of the real loading (best-estimate calculation) or penalized (conservative design).

- The calculation of the evolution in time of the stress tensor $S_{i j}(t)$ assuming a linear elastic material behaviour even for low cycles fatigue regime. In the next items, one will notice that the fatigue curve is always compatible with this assumption. Note that the stress tensor is evaluated at the points where the crack initiation is likely to occur.

- The use of the Tresca criterion to reduce the stress tensor $S_{i j}(t)$ to an equivalent scalar stress $S(t)$ is the recommended approach in the French standard for fatigue analysis in nuclear engineering (i.e. RCC-M [2]). This criterion has been chosen in the probabilistic approach for the sake of consistency with the deterministic design. 
- The extraction of stress cycles from the equivalent stress $S(t)$ using the Rainflow counting method (e.g. Amzallag et al. [3]).

- The choice of a fatigue design curve known as $S-N$ curve. This curve gives the number-of-cycles-to-failure $N(S)$ for a given stress amplitude $S$ and represents the fatigue strength of the material. It is initially based on strain-controlled fully reversed fatigue tests of small, polished specimens at room temperature in air. Then the strain amplitude is multiplied by the Young's modulus to obtain a pseudo-stress amplitude $S$. In this way, the obtained stress amplitude is compatible with the elastic material behaviour assumption in the stress calculation. The best-fit-curve to the experimental data is then lowered by a factor of 2 on stress amplitude $S$ or 20 on cycles number $N$, whichever was more conservative, to obtain the fatigue design curve (e. g. ASME [1]). The factors are intended to account for the differences and uncertainties in relating the fatigue lifetime of laboratory test specimens to those of the actual reactor components. The best-fit curve is expressed in terms of the Langer's equation of the form [4]:

$$
\ln N=A \ln \left(S-S_{D}\right)+B
$$

where $A, B$ and $S_{D}$ are parameters of the model. In particular, $S_{D}$ is the material endurance limit.

- The correction of the applied stress amplitude $S$ to account for the maximum mean stress effect using the following formula derived from the Goodman diagram:

$$
S^{\prime}=\frac{S_{u}-S_{y}}{S_{u}-S} S \text { for } S<S_{y}
$$


In this equation, $S$ is the applied stress amplitude, $S^{\prime}$ is the corrected value, $S_{u}$ is the ultimate tensile strength and $S_{y}$ is the yield strength. Both the fatigue design curve and the result from the Goodman correction are shown in Figure 2.

- The computation of the cumulative fatigue damage $D$ (also known as the usage factor) in a given period using the Miner's rule [5], which asserts that $D$ is given by:

$$
D=\sum_{i} \frac{n_{i}}{N\left(S_{i}\right)}
$$

where $n_{i}$ is the number of cycles of stress amplitude $S_{i}$ operating on the component and $N\left(S_{i}\right)$ is the number-of-cycles-to-failure at this stress amplitude.

The nuclear design code requires that the usage factor $D$ remains less than 1 . In practice, the thermal load history is supposed to be periodical in time and the usage factor $D_{\text {seq }}$ is computed on a single period. Then, the fatigue life-time of the component, expressed in terms of number of sequences, is given by $T_{d}=1 / D_{\text {seq. }}$.

\section{UNCERTAINTIES CHARACTERIZATION}

Two groups of uncertainties are identified in the description of the material thermal fatigue behaviour: those corresponding to the random nature of the input variables and those associated to the model error (e.g. Tovo [6]). The random input variables include the thermal loading variations, the thermo-mechanical parameters, the fatigue strength of laboratory test specimens and the factors relating the number-of-cycles-to-failure of laboratory test specimens to that of the actual component. The possible sources of

model error are associated to the theoretical assumptions of the modelling and the parameter estimations. 


\subsection{Thermal loading variations}

The fluid temperature in pipes is usually fluctuating and is hardly predictable with accuracy. It involves for example thermo-hydraulic phenomenon including turbulence that are still not well known and modelled. The temperature time history can be represented by a random process $\theta(t, \omega)$ where $t$ is the time and $\omega$ is associated to randomness. This notation means that for a constant $\omega=\omega_{0}, \theta\left(t, \omega_{0}\right)$ is a deterministic load history and for a constant $t=t_{0}, \theta(t, \omega)$ is a random variable (Figure 3). In this study, the load process is assumed to be Gaussian, stationary and ergodic since the considered service life-time is large enough. The choice of a stationary and ergodic random process is compatible with the little information available, but remains an essential assumption, even if it can be judged too strong.

\subsection{Thermo-mechanical parameters}

These parameters include geometrical dimensions, material properties and fluid/structure boundary conditions. The geometrical dimensions of a structure are not fixed but vary within the allowed manufacturing tolerance limits. Their probabilistic distribution is assumed to be lognormal so that they remain strictly positive. Their mean values are assumed to be equal to their respective nominal value. Their coefficients of variation may be obtained using the fact that the dimension under consideration falls within its tolerance limits with a probability of 95\% (e. g. Rusk and Hoffmann [7]). The material properties (e.g. Young's modulus, Poisson's ratio and thermal expansion coefficients) are also random in nature due to the material heterogeneity at the microscopic scale. Their probabilistic characterisation may require introducing random fields. However, the spatial variability of these properties is neglected in this study and 
they are consequently treated here as random variables with a lognormal distribution (which is common practice in literature). Their mean values are also assumed to be equal to their respective nominal value. Missing specific information on the problem under consideration, we assign to their coefficients of variation the value of $10 \%$ usually observed for steel (e. g. JCSS [8]). The dependence of these properties with respect to temperature is included in this modelling.

The fluid/structure boundary condition is characterised by the heat transfer coefficient. This coefficient, which depends on the fluid temperature and the flowing rate, is not well known. For this reason, it is treated as a random variable, whose distribution is assumed to be lognormal with a mean value fixed to its nominal value and a large coefficient of variation of $30 \%$ to take into account the big scatter on this parameter usually observed in model testing.

\subsection{Fatigue strength of laboratory test specimen}

Because of the large scatter observed in fatigue data at any given stress level, the number of cycles to failure at a given stress level is considered as a random variable. Many authors show that, for any stress level not closer to the endurance limit, the probabilistic distribution of the number of cycles to failure at that stress level can be regarded as lognormal, whose parameters depend on that stress level (e.g. Zhao et al. [9]). In this study we extend this assumption to the whole domain of stress amplitudes.

The characteristics of this distribution are estimated by a statistical analysis carried out on a database provided by Electricité de France (e. g. Sudret and Guédé [10]). This database consists on strain-controlled fully reversed fatigue tests of small polished 304 
and 316 austenitic steel specimens at room temperature in air. Only those tests which produce failure are considered, leading to a set of $Q=99$ values.

The lognormally distributed number of cycles to failure $N(S, \omega)$ at any stress level $S$ may be written as:

$$
\ln N(S, \omega)=\eta(S)+\varepsilon(S, \omega)
$$

where $\eta(S)$ is the mean value of the logarithm of $N$ and $\varepsilon(S, \omega)$ is a zero mean Gaussian random variable. Moreover, it is assumed that these variables (representing the scatter of $\ln N$ around its mean-value) are perfectly correlated. This assumption means that a given sample is good or bad with respect to fatigue life whatever the stress level applied. This assumption appears reasonable, although it is practically impossible to check, since each fatigue test is conducted up to the failure of the specimen. Thus Eq. (4) simplifies into:

$$
\ln N(S, \omega)=\eta(S)+\sigma(S) \xi(\omega)
$$

where $\xi(\omega)$ is a standard normal variable and $\sigma(S)$ is the standard deviation of $\ln N$. It is further assumed that $\eta(S)$ is expressed in terms of Langer's equation (Eq. (1)) and $\sigma(S)$ is proportional to $\eta(S)$ (i.e. the coefficient of variation $\delta$ of $\ln N(S, \omega)$ is constant).

$$
\sigma(S)=\delta \eta(S)
$$

Then the four parameters $A, B, S_{D}$ and $\delta$ are determined in a single shot using the method of maximum likelihood. Note that other assumptions on $\sigma(S)$ have been investigated in Sudret and Guédé [10], the most accurate being the one presented above. The method of maximum likelihood consists in maximizing the following function:

$$
L_{Q}\left(A, B, S_{D}, \delta\right)=\prod_{j=1}^{Q} \frac{1}{\sqrt{2 \pi} \sigma\left(S_{j}\right)} \exp \left[-\frac{1}{2}\left(\frac{\ln N_{j}-A \ln \left(S_{j}-S_{D}\right)-B}{\sigma\left(S_{j}\right)}\right)^{2}\right]
$$


or equivalently minimize the log-likelihood function:

$$
-2 \ln \left[L_{Q}\right]=\sum_{j=1}^{Q}\left[\ln \sigma^{2}\left(S_{j}\right)+\left(\frac{\ln N_{j}-A \ln \left(S_{j}-S_{D}\right)-B}{\sigma\left(S_{j}\right)}\right)^{2}\right]+Q \ln (2 \pi)
$$

In this equation, $Q$ is the number of data values $\left(S_{j}, N_{j}\right)$. The minimization problem is solved using MathCad routines [11]. The results are given in Table 1.

To complete the statistical analysis the assumption of normality of $\xi(\omega)$ is validated using the Kolmogorov test. This test consists in computing the maximal discrepancy $D_{K}$ between the empirical cumulative distribution $\hat{F}(\xi)$ and the hypothesis cumulative distribution $F(\xi)$ :

$$
D_{K}=\max |\hat{F}(\xi)-F(\xi)|
$$

The hypothesis regarding the theoretical distribution form is rejected if $D_{K}$ is greater than the critical value $D_{K}^{c}$ obtained from a table (e.g. Bain and Engelhardt, [12]) for a given confidence level. Applied to our database, the Kolmogorov test accepts the normality hypothesis with a confidence level of 95\% (Table 1).

\subsection{Specimens-to-structure passage factors}

The fatigue life tests are performed in idealized conditions in laboratory. However the actual structures are subject to varying environment and sometimes hard conditions. In the existing nuclear design code, the factors $\gamma^{N}=20$ and $\gamma^{S}=2$, which are introduced to relate the number-of-cycles-to-failure of laboratory test specimens to those of actual reactor components, can be interpreted as the product of two sub-factors:

$$
\gamma^{j}=\gamma_{\text {scatter }}^{j} \cdot \gamma_{\text {passage }}^{j} ; j=N, S
$$


where the subfactors $\gamma_{\text {scatter }}^{j}$ cover the scatter of the experimental data and the sub-factors $\gamma_{\text {passage }}^{j}$ account for the effects of the size, the surface finish and the industrial environment of the actual structure (e. g. Chopra and Shack [13]). Having noted that the scatter of the experimental data is already included in the probabilistic characterisation of the number-of-cycles-to-failure, the subfactors $\gamma_{\text {passage }}^{j}$ appear as conversion factors that must be applied to the fatigue lives of specimens to estimate the lives of actual reactor structures. However, the literature shows that these subfactors, which are more or less empirical, are still not well known (e.g. Framatome [14]). For this reason, they are regarded in this paper as random variables. Since it is possible to bound these factors, they are supposed to follow a Bêta distribution. The parameters of this Bêta distribution are yet to be determined from experiments carried out both on laboratory test specimen and operating structures and comparison thereof.

\subsection{Model parameter estimations}

Uncertainties in the estimates of model parameters are inherent to the use of mathematical functions to fit the available data. The estimators of these parameters are random variables. Thus, they must be accurately calculated and for each of them a confidence interval has to be computed.

\subsection{Theoretical assumptions of the modelling}

Simple models based on many hypotheses are used to describe the complex thermal fatigue process. These mathematical models can not account for all the possible factors that have an effect on the thermal fatigue behaviour of the structures and introduce therefore uncertainties in the modelling. As an example, the Miner's rule based on 
linear cumulated damage is not rigorously exact and the methods to deal with multiaxial fatigue cycles are still subject to discussion (e. g. You and Lee [15]). Moreover, in the nuclear design code the stress tensor is computed under the assumption of linear elastic material behaviour, whereas crack initiation is governed by plastic strain in the critical regions of the structure.

Yet, no model uncertainty is introduced in this study. Indeed, the objective of the present paper is to set up a reasonable probabilistic design against fatigue and not to find the idealized design.

\section{RANDOM FATIGUE DAMAGE}

Due to all the uncertainties in the model described in section 3 , the fatigue damage is a random variable. In this section, let us consider that only the fatigue strength of the material $N\left(S, \omega_{m}\right)$ and the thermal loading $\theta\left(t, \omega_{S}\right)$ are random, where $\omega_{S}$ and $\omega_{m}$ denote the randomness associated to the thermal loading and the material fatigue strength respectively, the other variables being considered as deterministic.

The thermal loading $\theta\left(t, \omega_{S}\right)$ is supposed to be Gaussian stationary process throughout the component service life. It is also assumed that the mechanical model relating the loading to the equivalent scalar stress $S\left(t, \omega_{S}\right)$ keeps the normality and the stationary state of the random process. This is the case when elastic material behaviour is imposed. Under this hypothesis, $S\left(t, \omega_{S}\right)$ is likewise a Gaussian stationary process. Let us consider $\left\{S_{i}\left(\omega_{S}\right)\right\}_{i=1}^{N_{c}}$ the set of stress amplitudes extracted from the equivalent stress by the Rainflow counting, where $N_{c}$, which is presumably random, denotes the total number of cycles throughout the actual component service life. Under the assumption of 
stationarity of $S\left(t, \omega_{S}\right)$ and for a large number of cycles, $N_{c}$ can be regarded as a constant (e.g. Tovo [6]). Finally, $\left\{S_{i}\left(\omega_{S}\right)\right\}_{i=1}^{N_{c}}$ is assumed to be a set of independent and identically distributed random variables with probability density function $f_{S}(S)$. The resulting random fatigue damage is expressed in two ways, namely by a continuous formulation and a discrete formulation.

\subsection{Continuous formulation}

Let $n\left(S, \omega_{S}\right) d S$ be the number of cycles of stress amplitudes in the elementary interval $[S ; S+d S]$ throughout the component service life. Since $N_{c}$ takes large values, the law of large numbers states that $n\left(S, \omega_{S}\right) d S$ is given by:

$$
n\left(S, \omega_{S}\right) d S=N_{c} f_{S}(S) d S
$$

Based on Miner's rule, the elementary damage associated to the cycles of stress amplitudes between $S$ and $S+d S$ is given by:

$$
d D\left(N_{c}, S, \omega_{S}, \omega_{m}\right)=\frac{N_{c} f_{S}(S) d S}{N\left(S, \omega_{m}\right)}
$$

The total damage is written as:

$$
D\left(N_{c}, S, \omega_{m}\right)=\int_{0}^{\infty} \frac{N_{c} f_{S}(S) d S}{N\left(S, \omega_{m}\right)}=N_{c} \mathrm{E}_{S}\left[\frac{1}{N\left(S, \omega_{m}\right)}\right]
$$

where $E_{S}[\cdot]$ is the mathematical expectation operator with respect to $\omega_{S}$. Note that in this formulation, the random damage contains no uncertainty related to the stress variations, since $\omega_{S}$ is removed by application of the expectation operator $E_{S}[\cdot]$. 


\subsection{Discrete formulation}

According to Miner's rule, the cumulative damage also reads:

$$
D\left(N_{c}, \omega_{S}, \omega_{m}\right)=\sum_{i=1}^{N_{c}} Y_{i}\left(\omega_{S}, \omega_{m}\right) \text { where } Y_{i}\left(\omega_{S}, \omega_{m}\right)=\frac{1}{N\left(S_{i}\left(\omega_{S}\right), \omega_{m}\right)}
$$

Having noted that $\left\{S_{i}\left(\omega_{S}\right)\right\}_{i=1}^{N_{c}}$ is a set of independent and identically distributed random variables, it is clear that $\left\{Y_{i}\left(\omega_{S}, \omega_{m}\right)\right\}_{i=1}^{N_{c}}$ is, likewise, a set of independent and identically distributed random variables. Let $\mu_{Y}\left(\omega_{m}\right)=\mathrm{E}_{S}\left[Y_{i}\left(\omega_{S}, \omega_{m}\right)\right] \quad$ and $\sigma_{Y}^{2}\left(\omega_{m}\right)=\operatorname{var}_{S}\left[Y_{i}\left(\omega_{S}, \omega_{m}\right)\right]$ be the mean and the variance of the $Y_{i}$ 's with respect to $\omega_{S}$. Since $N_{c}$ is large enough, the central limit theorem states that the cumulative damage tends to be normally distributed with mean value $N_{c} \mu_{Y}\left(\omega_{m}\right)$ and standard deviation $\sigma_{Y}\left(\omega_{m}\right) \sqrt{N_{c}}$. Thus, for a sufficiently large value of $N_{c}$, the cumulative random damage is approximated by:

$$
D\left(N_{c}, \omega_{S}, \omega_{m}\right) \approx N_{c} \mu_{Y}\left(\omega_{m}\right)\left[1+\frac{\sigma_{Y}\left(\omega_{m}\right)}{\mu_{Y}\left(\omega_{m}\right) \sqrt{N_{c}}} \xi\left(\omega_{S}\right)\right]
$$

where $\xi\left(\omega_{S}\right)$ is a standard Gaussian variable. When $N_{c}$ tends to infinity, the factor $\sigma_{Y}\left(\omega_{m}\right) / \mu_{Y}\left(\omega_{m}\right) \sqrt{N_{c}}$ vanishes, and the damage becomes:

$$
D\left(N_{c}, \omega_{S}, \omega_{m}\right) \approx N_{c} \mu_{Y}\left(\omega_{m}\right)=N_{c} \mathrm{E}_{S}\left[\frac{1}{N\left(S, \omega_{m}\right)}\right]
$$

As a consequence, Eq. (16) appears identical to Eq. (13) in this limit. As a conclusion, under the assumption of large and constant total number of cycles, the two formulations are equivalent. However, the discrete formulation explicitly contains the discrepancy to the limit for medium $N_{c}$. 


\section{IMPLEMENTATION OF THE THERMAL FATIGUE ASSESSMENT IN STRUCTURAL RELIABILITY}

The general method used here to assess reliability of a structure submitted to thermal fatigue consists in incorporating in the modelling of the thermal fatigue behaviour all kinds of uncertainties described in section 3. The objective of the analysis is to compute the failure probability for a given service life-time. The proposed probabilistic approach of thermal fatigue design is based on the theory and methods of structural reliability, which are briefly recalled below.

\subsection{Review of structural reliability methods}

Structural reliability analysis is based on (e. g. Ditlesven and Madsen [16]):

- The choice of a stochastic model for the set $\mathbf{X}(\omega)$ of input random variables (i.e. marginal densities and correlation matrix, or better, the joint density function $\left.f_{\mathrm{X}}(\mathbf{x})\right)$

- The definition of a failure scenario through the determination of a limit state function $G(\mathbf{X})$ which splits the safe domain (defined by $G(\mathbf{X})>0$ ) from the failure domain (defined by $G(\mathbf{X}) \leq 0$ ). The boundary defined by $\{\mathbf{X}$ such that $G(\mathbf{X})=0\}$ is called the limit state surface.

The failure probability reads:

$$
P_{f}=\int_{G(\mathbf{X}) \leq 0} f_{\mathbf{X}}(\mathbf{x}) d \mathbf{x}
$$

This probability may be evaluated using the First Order Reliability Method (FORM), whose main steps are: 
1. A probability preserving transformation of the set of variables $\mathbf{X}(\omega)$ in the physical space to a set of uncorrelated standard normal variables $\mathbf{U}(\omega)$ in the socalled standardized normal space (Figure 4). In general, the Rosenblatt transformation or the Nataf transformation are used (e. g. Melchers [17]). Note that in the standardized normal space, the limit state function is denoted by $H(\mathbf{U})$.

2. The determination, in the standardized space, of the design point $\mathbf{u}^{*}$ (i.e. the point on the limit-state surface with maximum value of the probability density function) solving the optimization problem using mathematical programming methods:

$$
\left\|\mathbf{u}^{*}\right\|=\min _{\mathbf{u}}\|\mathbf{u}\| \text { under the constraint } H(\mathbf{U}) \leq 0
$$

The distance from the origin of the standardized space to the design point is the reliability index, denoted by $\beta$.

3. The estimation of the failure probability using a linear approximation of the limit-state surface at the design point. Due to the rotational symmetry of the standardized space, the failure probability approximation is analytic:

$$
P_{f} \approx \Phi(-\beta)
$$

where $\Phi(\cdot)$ is the standard normal integral.

In addition to the failure probability, the FORM method gives the importance factors, which make it possible to classify the variables according to their weight in the reliability. These factors reads $\alpha_{i}^{2}$ for each random input variable $U_{i}$, where the $\alpha_{i}$ 's are the direction cosines of the unit normal vector $\vec{\alpha}$ at the design point to the limitstate surface (Figure 5). 
When the limit state function is strongly non-linear, the FORM method becomes less accurate. In this case, the second order reliability method (SORM) is an alternative to improve the accuracy of FORM. It consists in fitting a quadratic surface centered at the design point. Then the failure reliability is estimated by the probabilistic content of the quadratic domain approximating the failure region and depends mainly on the curvatures at the design point. In particular, the asymptotic Breitung's formula [18] may be used:

$$
P_{f}=\Phi(-\beta) \prod_{i=1}^{n-1}\left(1+\beta \kappa_{i}\right)^{-1 / 2}
$$

where $n$ is the number of random input variables and $\kappa_{i}$ is the ith principal curvature of the limit-state surface at the design point.

The failure probability may also be calculated using simulation methods, in particular the importance sampling method, which is a Monte-Carlo simulation targeted at the design point. This method allows to obtain accurate values of the failure probability for a relatively low number of simulations (e.g. 1000 simulations). Let us write the probability integral in Eq. (17) using the indicator function of the failure domain in the standard normal space $I[H(\mathbf{U}) \leq 0]$ :

$$
P_{f}=\int_{R^{n}} I[H(\mathbf{U}) \leq 0] \frac{\varphi_{n}(u)}{\psi(u)} \psi(u) d u
$$

In this expression, $\varphi_{n}$ is the multinormal density function and $\psi$ is termed the "importance sampling" distribution (e.g. the multinormal density function centered at the design point with a unit standard deviation). From Eq. (21), the failure probability is regarded as the expectation of a function of $\mathbf{u}$ with respect to the importance sampling distribution: 


$$
P_{f}=\mathrm{E}_{\psi}\left[I[H(U) \leq 0] \frac{\varphi_{n}(u)}{\psi(u)}\right]
$$

Then, Eq. (22) can be estimated by Monte-Carlo simulation using $K$ samples:

$$
P_{f}=\frac{1}{K} \sum_{i=1}^{K} I\left[H\left(u^{(i)}\right) \leq 0\right] \frac{\varphi_{n}\left(u^{(i)}\right)}{\psi\left(u^{(i)}\right)}
$$

Since the importance sampling method approaches the exact failure probability for large values of $K$, it can be used to check the FORM and SORM approximations. These three methods are illustrated in Figure 5.

In practice, the computation of the failure probability using the above methods is carried out by coupling the stochastic model, which is the probabilistic characterisation of the input random variables, and a mechanical model, defined as the procedure to compute the limit state function (e. g. Lemaire [19]). The structural reliability methods are implemented in the software PHIMECA Soft ${ }^{\circledR}$ [20] which is used in the present analysis.

\subsection{Thermal fatigue reliability assessment}

Let us denote by $\mathbf{x}$ a sample of $\mathbf{X}$, the vector of the random input variables. Given $N_{0}$, the target number-of-cycles in service of the structure under consideration, the failure occurs when the random number-of-cycles-to-failure $N(\mathbf{x})$ is less than $N_{0}$. The limit state function associated with this problem therefore reads:

$$
G(\mathbf{x})=N(\mathbf{x})-N_{0}
$$

An alternative and equivalent expression is given by:

$$
G(\mathbf{x})=1-D\left(T_{0}, \mathbf{x}\right)
$$


where $D\left(T_{0}, \mathbf{x}\right)$ is the total fatigue damage produced during the target life-time $T_{0}$. Let us assume that the joint PDF $f_{\mathbf{X}}(\mathbf{x})$ of $\mathbf{X}$ is known. In practice, only the knowledge of the marginal distributions of each input variables and the correlation matrix is needed. Two dual approaches of the reliability analysis are defined, respectively in the time domain and in the frequency domain.

\subsubsection{Reliability analysis in the time domain}

Let $\mathbf{x}$ be a sample of the input random variables of the problem under consideration. In particular, a sample of the thermal loading process is given by a time history on the target service life-time $T_{0}$. The thermal stress tensor $S_{i j}(\mathbf{x}, t)$ is evaluated at a probable critical location on the structure. Then, Tresca's criterion is used to reduce the stress tensor to an equivalent scalar stress $S(\mathbf{x}, t)$ on which the Rainflow counting method is applied to find the cycles amplitudes $\left\{S_{k}(\mathbf{x}), k=1, \ldots, N_{c}\right\}$, where $N_{c}$ is the total numberof-stress-cycles. From the fatigue strength model, the admissible number of cycles $N^{\text {struct }}\left(S_{k}(\mathbf{x}), \mathbf{x}\right)$ associated to each cycle amplitude $S_{k}(\mathbf{x})$ is determined. Finally, the total fatigue damage for the target service life-time in $T_{0}$ is computed as follows:

$$
D\left(T_{0}, \mathbf{x}\right)=\sum_{k=1}^{N_{c}} \frac{1}{N^{\text {struct }}\left(S_{k}(\mathbf{x}), \mathbf{x}\right)}
$$

Note that the total fatigue damage expression in Eq. (26) corresponds to its discrete formulation (section 4.2).

If the thermal load was considered as a Gaussian random process, it is noted that it will be discretized, i.e. represented by a finite set of random variables. See for example Shinozuka and G. Deodatis [21] or Zhang and Der Kiureghian [22]. Then, these random variables can be considered as input data of the problem. Each realization of these 
variables produces a trajectory of the temperature process that is included in the whole procedure.

In conclusion, when the probability distributions of all random variables are defined, it is possible to compute the limit state function from a sample of the random input variables using the scheme described above (Figure 6) which is the probabilistic assessment in the time domain of the deterministic model (Figure 2). It is therefore possible to link the stochastic model to the mechanical model, then to assess reliability of the structure submitted to thermal fatigue. This approach seems natural since the limit state function is computed with the currently codified design procedure [2].

\subsubsection{Reliability analysis in the frequency domain}

This approach is based on spectral methods of fatigue life-time prediction for mechanical components submitted to a stationary load random process. These methods aim at evaluating the fatigue life-time directly from the spectral properties of the stress random process resulting from the load random process. Let $W_{\theta}(\lambda)$ be the power spectral density function (PSD) of the thermal load process ( $\lambda$ is the frequency in radians per second). We successively compute the PSD of the stress random process, the PDF of the stress amplitudes and the total fatigue damage.

\section{a/ Power spectral density function of the stress random process}

The stresses in the structure are computed under the assumption of a linear material behaviour, as is common practice in the nuclear design code. The problem is therefore described as a linear physical system with a single input, given by the random thermal load $\theta(t, \omega)$, and a single output, given by the stress $S(t, \omega)$ in the structure under 
consideration. When the random thermal load is assumed to be stationary, the random stress process is also stationary due to linearity of the system and the PSD of the stress process $W_{s}(\lambda)$, is obtained by (e. $g$. Preumont [23]):

$$
W_{S}(\lambda)=|T F(\lambda)|^{2} W_{\theta}(\lambda)
$$

where $\operatorname{TF}(\lambda)$ denotes the transfer function of the system, given by the Fourier transform of the impulse response $z(t)$ :

$$
T F(\lambda)=\int_{-\infty}^{\infty} z(\tau) e^{-j \lambda \tau} d \tau
$$

In the case of a multi-axial state of stress, the transfer function becomes a matrix and the multi-axial fatigue criteria formulated in the frequency domain can be used to compute the PSD of the scalar equivalent stress. The literature gives the spectral formulation of some multi-axial fatigue criteria, in particular those based on stress invariants or those using the critical plane approach (e. g. Pitoiset and Preumont, [24]).

b/ Probability distribution of stress amplitudes

The probability density function of the stress amplitudes is computed using the spectral moments of the scalar equivalent stress. The spectral moment $m_{i}$ of order $i$ is defined as follows:

$$
m_{i}=\int_{-\infty}^{\infty}|\lambda|^{i} W_{S}(\lambda) d \lambda
$$

In this analysis, the empirical Dirlik’s formula is used [25]. It is based on four spectral moments of the equivalent stress, namely $m_{0}, m_{1}, m_{2}$ and $m_{4}$. By this formula, the number of Rainflow cycles with range in the interval $[\Delta S ; \Delta S+d \Delta S$ ] is given by:

$$
n(\Delta S)=\mathbf{E}\left[M_{T}\right] T_{0} p(\Delta S)
$$


where $T_{0}$ is the service life of the structure, $\mathbf{E}\left[M_{T}\right]$ is the mean number of peaks per unit time and reads:

$$
\mathbf{E}\left[M_{T}\right]=\frac{1}{2 \pi} \sqrt{\frac{m_{4}}{m_{2}}}
$$

and $p(\Delta S)$ is the probability to extract one cycle with range in the interval $[\Delta S ; \Delta S+d \Delta S$ ]. This probability is given by:

$$
p(\Delta S)=\frac{1}{2 \sqrt{m_{0}}}\left[\frac{D_{1}}{Q} e^{-\frac{Z}{Q}}+\frac{D_{2} Z}{R^{2}} e^{-\frac{Z^{2}}{2 R^{2}}}+D_{3} Z e^{-\frac{Z^{2}}{2}}\right]
$$

where:

$$
\begin{aligned}
& D_{1}=\frac{2\left(x_{m}-\gamma^{2}\right)}{1+\gamma^{2}} ; \quad D_{2}=\frac{1-\gamma-D_{1}+D_{2}}{1-R} ; D_{3}=1-D_{1}-D_{2} ; \\
& x_{m}=\frac{m_{1}}{m_{0}} \sqrt{\frac{m_{2}}{m_{4}}} ; \gamma=\frac{m_{2}}{\sqrt{m_{0} m_{4}}} ; R=\frac{\gamma-x_{m}-D_{1}^{2}}{1-\gamma-D_{1}+D_{1}^{2}} ; \\
& Q=\frac{1.25\left(\gamma-D_{3}-D_{2} R\right)}{D_{1}} ; Z=\frac{\Delta S}{2 \sqrt{m_{0}}} .
\end{aligned}
$$

Figure 7 shows the goodness of fit of the Dirlik's formula to the Rainflow cycles for two uniformly distributed PSD, respectively with narrow and large bandwidth.

\section{c/ Total fatigue damage}

From the Dirlik's formula in Eq. (33), it is easy to show that the PDF of the stress amplitudes $S=\Delta S / 2$ reads:

$$
f_{S}(S)=\frac{1}{2} p(\Delta S)=\frac{1}{2} p(2 S)
$$

Thus, the number of Rainflow cycles, whose amplitudes belong to the interval $[S ; S+d S$ ] is given by: 


$$
n(S, \mathbf{x}) d S=\mathbf{E}\left[M_{T}\right] T_{0} f_{S}(S, \mathbf{x}) d S
$$

In the latter equation, $\mathbf{x}$ denotes the realization of the input random variables describing the mechanical model. The value of all parameters entering Eq. (34) (see Eq. (33)) depend indeed implicitly on these input parameters $\mathbf{x}$.

Using Miner's rule, the elementary damage $d D_{T}(S, \mathbf{x})$ caused by the stress cycles with amplitudes in the interval $[S ; S+d S$ ] reads:

$$
d D_{T}(S, \mathbf{x})=\frac{n(S, \mathbf{x}) d S}{N^{\text {struct }}(S, \mathbf{x})}=\frac{\mathbf{E}\left[M_{T}\right] T_{0} f_{S}(S, \mathbf{x}) d S}{N^{\text {struct }}(S, \mathbf{x})}
$$

where $N^{\text {struct }}(S, \mathbf{x})$ represents the fatigue strength of the structure under consideration and is defined as the number-of-cycles-to-failure under cyclic stress with constant amplitude $S$. The total fatigue damage is obtained by integrating Eq.(32) on the variation domain of $S$ :

$$
D_{T}(\mathbf{x})=\int_{0}^{\infty} \frac{n(S, \mathbf{x}) d S}{N^{\text {struct }}(S, \mathbf{x})}=T_{0} \int_{0}^{\infty} \frac{\mathbf{E}\left[M_{T}\right] f_{S}(S, \mathbf{x}) d S}{N^{\text {struct }}(S, \mathbf{x})}
$$

Note that, the fatigue damage is computed by means of its continuous formulation (section 4.1) in this case.

In conclusion, when a sample of the input random variables is generated, it is possible to compute the associated value of the limit state function using the procedure described above (Figure 8) which is the probabilistic assessment in the frequency domain of the deterministic model (Figure 2). Therefore, this procedure can serve as a mechanical model to be coupled to the stochastic model to assess the failure probability against thermal fatigue. 


\section{APPLICATION}

\subsection{Problem statement}

Consider a free end pipe subjected to variable temperature due to water flow (Figure 9). We want to perform a complete reliability analysis of this structure including all the uncertainties in the fatigue behaviour and in the mechanical model. The statistical properties of the input random variables are given in the Table 2 . The objective is to compute the failure reliability and the associated reliability index for a given service life-time. Beside the failure reliability, the sensitivity of the result to each random variable is estimated. In this analysis we want to compare the results given by the time domain and the frequency domain approach respectively. Both high cycle fatigue (HCF) and low cycle fatigue (LCF) regimes are investigated.

\subsection{Thermal loading model}

The thermal load is assumed to be random and represented by a random Gaussian stationary process. In this example two random thermal loading are considered. They are represented as Gaussian pseudo-white noise random processes with zero central frequency and different cut-off frequencies. The first one is denoted $\theta_{1}(t, \omega)$ and its cutoff frequency is equal to $f_{1}=20 \mathrm{~Hz}$. The second one, $\theta_{2}(t, \omega)$, has a cut-off frequency $f_{2}=5 \mathrm{~Hz}$. Their respective mean value is $130^{\circ} \mathrm{C}$ and their standard deviation is equal to $20^{\circ} \mathrm{C}$. Both thermal loading models are illustrated respectively in Figures 10 and 11 .

For the analysis in the frequency domain, the random loading is represented by its power spectral density, denoted by $W_{1}(\lambda)$ and $W_{2}(\lambda)$ respectively. For the analysis in the time domain, the loading is given by a deterministic temperature sequence, since the 
damage is not affected by the loading variability for high number of cycles (see section 4). In order to compare both approaches, the temperature sequences used in the time domain analysis are simulated from their respective PSD. For cost reduction issues, these time-histories are considered periodical with periodicity equal $360 \mathrm{~s}$. Thus, we implicitly assume that the time-history of the thermal loading calculated on that period is representative of the real thermal load. Practically, the temperatures trajectory is discretized into $2^{14}=16384$ points.

Under the thermal loads $\theta_{1}(t, \omega)$ and $\theta_{2}(t, \omega)$ described above, the pipe operates in the HCF regime. In order to investigate the LCF regime, the stress amplitudes are raised by a factor $k$ in the second part of the study. This factor $k$ may be interpreted as a stress concentration factor to account for the presence a welded joint or a geometrical irregularity. In this numerical application, the factor $k$ is artificially given a value of 4 , intentionally exaggerated to emphasize the required phenomenon (i.e. being in the LCF domain)

\subsection{Mechanical model}

The pipe is assumed to be thin (e $\left.<<R_{i}\right)$ and uniformly loaded onto its inner surface. Under these assumptions, the temperature field in the thickness is solution of a one dimension unsteady heat conduction equation:

$$
\frac{\partial \theta(t, x)}{\partial t}=C \frac{\partial^{2} \theta(t, x)}{\partial x^{2}}
$$

where $C=L / \rho C_{p}$ is the thermal diffusivity of the material, $L$ is the conductivity and $\rho C_{p}$ is the heat capacity. The boundary conditions, which account for a perfectly 
insulate outer surface and a standard heat transfer model with the fluid, read respectively:

$$
\left\{\begin{array}{c}
\left.\frac{\partial \theta(t, x)}{\partial x}\right|_{x=R_{i}+e}=0 \\
-\left.L \frac{\partial \theta(t, x)}{\partial x}\right|_{x=R_{i}}=h\left[\theta\left(t, x=R_{i}\right)-\theta_{f}\right]
\end{array}\right.
$$

where $h$ is the heat transfer coefficient and $\theta_{f}$ is the fluid temperature.

Once the temperature profile in the thickness is obtained, the stress tensor is given by the equilibrium equation:

$$
\operatorname{div}\left(S_{i j}\right)=0
$$

under the following boundary conditions:

$$
\left\{\begin{array}{cc}
S_{r r}\left(x=R_{i}\right)=0 & \begin{array}{c}
\text { (zero inner pressure) } \\
\text { (free outer border) }
\end{array} \\
S_{r r}\left(x=R_{i}+e\right)=0 & \text { (free ends) } \\
\int_{R_{i}}^{R_{i}+e} x S_{r r}(x) d x=0 &
\end{array}\right.
$$

Due to the assumption of a free end thin pipe, the generalized plane strain conditions are applied (i.e. strain component $\varepsilon_{z z}$ is regarded as a constant throughout the pipe thickness and is an unknown parameter of the problem).

When time domain analysis is considered, the stress field in the thickness of the pipe is computed numerically using the finite difference code OSTAND developed by EDF $R \& D$, while it is obtained by an analytical transfer function in the frequency domain analysis (e. g. Musi and Beaud [26]). In both analyses, the two stress components $\sigma_{\theta \theta}$ and $\sigma_{z z}$ are equal at the inner wall, all other components being zero. Thus, the equivalent scalar stress (based on Tresca criterion), from which the stress cycles amplitudes are obtained, is $\sigma_{\theta \theta}=\sigma_{z Z}$. 


\subsection{Results}

The reliability analysis is carried out for 8 case studies. Indeed, for both thermal loads $\theta_{1}(t, \omega)$ and $\theta_{2}(t, \omega)$, HCF and LCF (using the stress factor $k$ ) modes are investigated, each of them being treated in the time and the frequency domain. Note that in the HCF domain the reliability is computed on a target service-life-time $N_{0}=10000$ temperature sequences of $360 \mathrm{~s}$ (i.e. $10^{7}$ stress cycles), while in the LCF domain it is performed on a target service-life-time $N_{0}=10$ temperature sequences (i.e. $10^{4}$ stress cycles).

For each case, the failure reliability is calculated using FORM, SORM and importance sampling methods. The generalized reliability indices $\beta=-\Phi^{-1}\left(P_{f}\right)$ given by these three methods are reported in Table 3. Concerning the importance factors, their values in both time and frequency domain, which are very closed, are shown in Figure 12 and 13. The complete results of this analysis are reported in Guédé [27].

In each case, the reliability indices provided by the various methods are very close. The relative differences between FORM approximations and importance sampling results vary between $0 \%$ and $5 \%$. Method FORM thus provides a very good approximation of the index of reliability.

From the reliability index values shown in Table 3, one can see that the frequency domain approach is always more conservative than time domain one (i.e. $\beta$ is always smaller). This is due to the fact that the frequency domain approach, which, unlike the time domain approach, incorporates the loading randomness and this includes more uncertainties. A larger difference between the respective reliability indices of both approaches (i.e. approximately 20\%) is observed for the first temperature model $\theta_{1}$, while a negligible difference appears for the second loading model $\theta_{2}$. The large 
difference in the first case does not question the equivalence of both approaches yet. It comes from the fact that both discrete and continuous formulations of the random damage are no longer equivalent because of a too low number of damaging stress cycles (i.e. 2 - 4 damaging stress cycles for a number of 4000 extracted cycles) extracted from the loading time history $\theta_{1}(t)$ throughout the chosen time duration of the temperatures sequence (i.e. 360 s). The observed difference should decrease for a higher period, which was not checked is the present work for reasons of computing time.

The importance factors shown in Figures 12 and 13 reveal that the scatter in fatigue strength and the heat transfer coefficient are the most significant variables in the thermal fatigue reliability. On the other hand, the effect of the heat transfer coefficient decreases in the LCF regime, since it acts directly on the variations of the stress cycles number, which is not that significant for the fatigue damage in the LCF domain.

The stress amplitude assessment via the passage factor $\gamma_{\text {passage }}^{S}$ is significant for the reliability only in the HCF domain where it is applied. On the other hand, the effect of the life-time reduction factor $\gamma_{\text {passage }}^{N}$ remains negligible in the LCF where it is applied. Indeed, even with the majoration of the stress amplitude by a high $k$ factor, the pipe does not operate exclusively in the LCF regime, but there are still many stress amplitudes in the HCF domain. The effect of $\gamma_{\text {passage }}^{N}$ should be risen in the case that only LCF regime is considered.

The Young's modulus and the thermal expansion coefficient are the most important thermal parameters for the fatigue reliability, due to the assumption of material linear elastic behaviour. Moreover, because of their symmetrical role in the mechanical model, their respective weights are equal. They may therefore be grouped into a single random variable, namely the product $E \alpha$. 


\section{CONCLUSIONS}

In the present work, a probabilistic approach for the assessment of nuclear components submitted to thermal fatigue is set up, making it possible to take account for all the uncertainties that affect the thermal fatigue behaviour. The procedure is applied to a pipe subjected to a random thermal loading. It is shown that it is possible to perform a complete reliability analysis against thermal fatigue, and to compute the reliability indices and the sensitivity factors with respect to any variation in each input variable. It is important to note that the proposed approach does not depend on the models used to describe thermal fatigue behaviour of the structure, and it could be adjusted for any other more accurate modelling.

It is also shown that the assessment of thermal fatigue reliability can be carried out equivalently in the time and frequency domain under the assumption of stationary Gaussian loading and large number of damaging stress cycles. However, it should be noted that the frequency domain approach can be used only for stationary random loading with an elastic material behaviour. On the other hand, the application of the time domain approach may be extended to non-stationary random loading, provided the non-stationary process is correctly discretized.

\section{REFERENCES}

[1] ASME. Criteria of the ASME boiler and pressure vessel code for design and analysis in sections III and VIII, division. Technical report, The American Society of Mechanical Engineers, New York, 1969.

[2] Collectif. Règles de Conception et de Construction des Matériels des Ilots Nucléaires (RCCM). AFCEN, Paris La Défense, 2000.

[3] Amzallag, C., Gerey, J. P., Robert, J. L. and Bahuaud, J. Standardization of the rainflow counting method for fatigue analysis. Int. J. of Fatigue 1994;16:287293. 
[4] Langer, B. F. Design of pressure vessels for low-cycle fatigue. ASME J. of Basic Eng. 1962;84:389-402.

[5] Miner, M. A. Cumulative damage in fatigue. J. Appl. Mech. 1945;12:A-159.

[6] Tovo, R. On the fatigue reliability evaluation of structural components under service loading. Int. J. of Fatigue. 2001;23:587-598.

[7] Rusk, D. T. and Hoffmann, P. C. Component geometry and material property uncertainty model for probabilistic strain-life fatigue predictions. In $6^{\text {th }}$ Joint FAA/DoD/NASA Aging Aircraft Conference, 2002.

[8] JCSS. Probabilistic model code. The Joint Committee on Structural Safety, 2001.

[9] Zhao, Y.-X., Gao, Q. and Wang, J. N. An approach for determining an appropriate assumed distribution of fatigue life under limited data. Reliability, Engineering and System Safety. 2000;67:1-7.

[10] Sudret, B. and Guédé, Z. Probabilistic assessment of thermal fatigue in nuclear components. Nucl. Eng. Des. 2005;235:1819-1835.

[11] Mathcad. User's Manual, Math soft Edition 2001.

[12] Bain, L. J. and Engelhardt, M. Introduction to Probability and Mathematical Statistics. Duxbury Classic series, Second edition, 2000.

[13] Chopra, O. K. and Shack, W. J. Low-cycle fatigue of piping and pressure vessel steels in LWR environments, Nuc. Eng. Des. 1998;184:49-76.

[14] Framatome. Re-evaluation of fatigue analysis criteria. Technical report EE/S 98.317, 1998.

[15] You, B. R. and Lee, S.B. A critical review on multiaxial fatigue assessments of metals. Int. J. of Fatigue 1996;18(4):235-244.

[16] Ditlevsen, O. and Madsen, H. O. Structural reliability methods, Wiley, 1996.

[17] Melchers, R. E. Structural reliability analysis and prediction. Wiley, 1999.

[18] Breitung, K. Asymptotic approximations for multinormal integrals. J. Eng. Mech. ASCE 1984 ;110(3):357-466.

[19] Lemaire, M. Fiabilité des structures - couplage mécano-fiabiliste statique. Hermes Science Publication 2005, Reliability of Structures, English translation, 2006. 
[20] PHI-MECA Engineering S.A. User’s Manual, PHIMECA Soft. 2003.

[21] Shinozuka, M. and Deodatis, G.. Simulation of stochastic process by spectral representation. Appl. Mech. Review 1991 ;44:191-204.

[22] Zhang, Y., and Der Kiureghian, A. Dynamic response sensitivity of inelastic structures, Comp. Methods Appl. Mech. Eng. 1993 ;108(1) :23-36.

[23] Preumont, A. Random vibration and spectral analysis. Kluwer Academic Publishers 1994.

[24] Pitoiset, X. and Preumont, A. Spectral methods for multiaxial random fatigue analysis of metallic structures. Int. J. of Fatigue $2000 ; 22: 541-550$.

[25] Dirlik, T. Application of computers in fatigue analysis. PhD thesis, University of Warwick, 1985.

[26] Musi, S. and Beaud, F. An analytical model for thermal fatigue crack initiation and propagation in mixing zones of piping systems. PVP - Fatigue, Fracture and Damage Analysis, 2003 ; 461 :111-118.

[27] Guédé, Z. Approche probabiliste de la durée de vie des structures sollicitées en fatigue thermique. $\mathrm{PhD}$ thesis. Université Blaise Pascal de Clermont-Ferrand France, 2005.

\section{NOMENCLATURE}

\begin{tabular}{|l|l|}
\hline$\omega, \omega_{m}, \omega_{S}$ & variables associated with randomness \\
\hline$t$ & time \\
\hline$\lambda$ & frequency \\
\hline$\theta(t, \omega)$ & random thermal loading history \\
\hline$S_{i j}(t)$ & stress tensor \\
\hline$S(t, \omega)$ & random equivalent scalar stress by Tresca criterion \\
\hline$S$ & stress amplitude \\
\hline$S^{\prime}$ & corrected value of the stress amplitude by the Goodman diagram \\
\hline$S_{u}$ & ultimate tensile strength \\
\hline$S_{y}$ & yield strength \\
\hline$E$ & Young's modulus \\
\hline$v$ & Poisson ratio \\
\hline$\alpha$ & thermal expansion \\
\hline$L$ & thermal conductivity \\
\hline$\rho C_{p}$ & specific heat capacity \\
\hline$h$ & heat transfer coefficient \\
\hline$\gamma_{\text {scatter }}^{N}, \gamma_{\text {passage }}^{N}$ & $\begin{array}{l}\text { subfactors for data scatter and for passage specimen to structure in } \\
\text { the low cycles fatigue domain }\end{array}$ \\
\hline
\end{tabular}




\begin{tabular}{|l|l|}
\hline$\gamma_{\text {scatter }}^{S}, \gamma_{\text {passage }}^{S}$ & $\begin{array}{l}\text { subfactors for data scatter and for passage specimen to structure in } \\
\text { the high cycles fatigue domain }\end{array}$ \\
\hline$S_{D}$ & endurance limit \\
\hline$A, B$ & parameters of the $S$ - $N$ curve model \\
\hline$D$ & cumulative fatigue damage \\
\hline$N(S, \omega)$ & $\begin{array}{l}\text { random number of cycles of a specimen under constant stress } \\
\text { amplitude repeated loading }\end{array}$ \\
\hline$N_{0}$ & target number of cycles in service \\
\hline$T_{0}$ & target fatigue lifetime in service \\
\hline$\eta(S)$ & best-fit curve to the fatigue tests data \\
\hline$\sigma(S)$ & standard deviation of ln $N$ \\
\hline$\delta$ & coefficient of variation of ln $N$ \\
\hline$D_{K}$ & variable of the statistical Kolmogorov test \\
\hline$D_{K}^{c}$ & critical value of $D_{K}$ in the statistical Kolmogorov test \\
\hline$f_{S}(S)$ & probability density function of the stress amplitude \\
\hline$W_{\theta}(\lambda)$ & power spectral density function of the thermal load process \\
\hline$W_{S}(\lambda)$ & power spectral density function of the equivalent stress process \\
\hline$T F(\lambda)$ & transfer function \\
\hline$f_{\mathbf{X}}(\mathbf{x})$ & joint probability density function of the input variables \\
\hline$G(\mathbf{x})$ & limit-state function \\
\hline$H(\mathbf{u})$ & limit-state function in the standardized normal space \\
\hline $\mathbf{u}^{*}$ & design point in the standardized normal space \\
\hline$P_{f}$ & failure probability \\
\hline$\beta$ & reliability index \\
\hline &
\end{tabular}

Operators

\begin{tabular}{|l|l|}
\hline$\Phi(\cdot)$ & standard normal cumulative distribution function \\
\hline$I[\cdot]$ & indicator function \\
\hline $\operatorname{div}(\cdot)$ & divergence operator \\
\hline
\end{tabular}

Abbreviations

\begin{tabular}{|l|l|}
\hline HCF & high cycles fatigue \\
\hline LCF & low cycles fatigue \\
\hline FORM & first order reliability method \\
\hline SORM & second order reliability method \\
\hline IS & importance sampling method \\
\hline
\end{tabular}




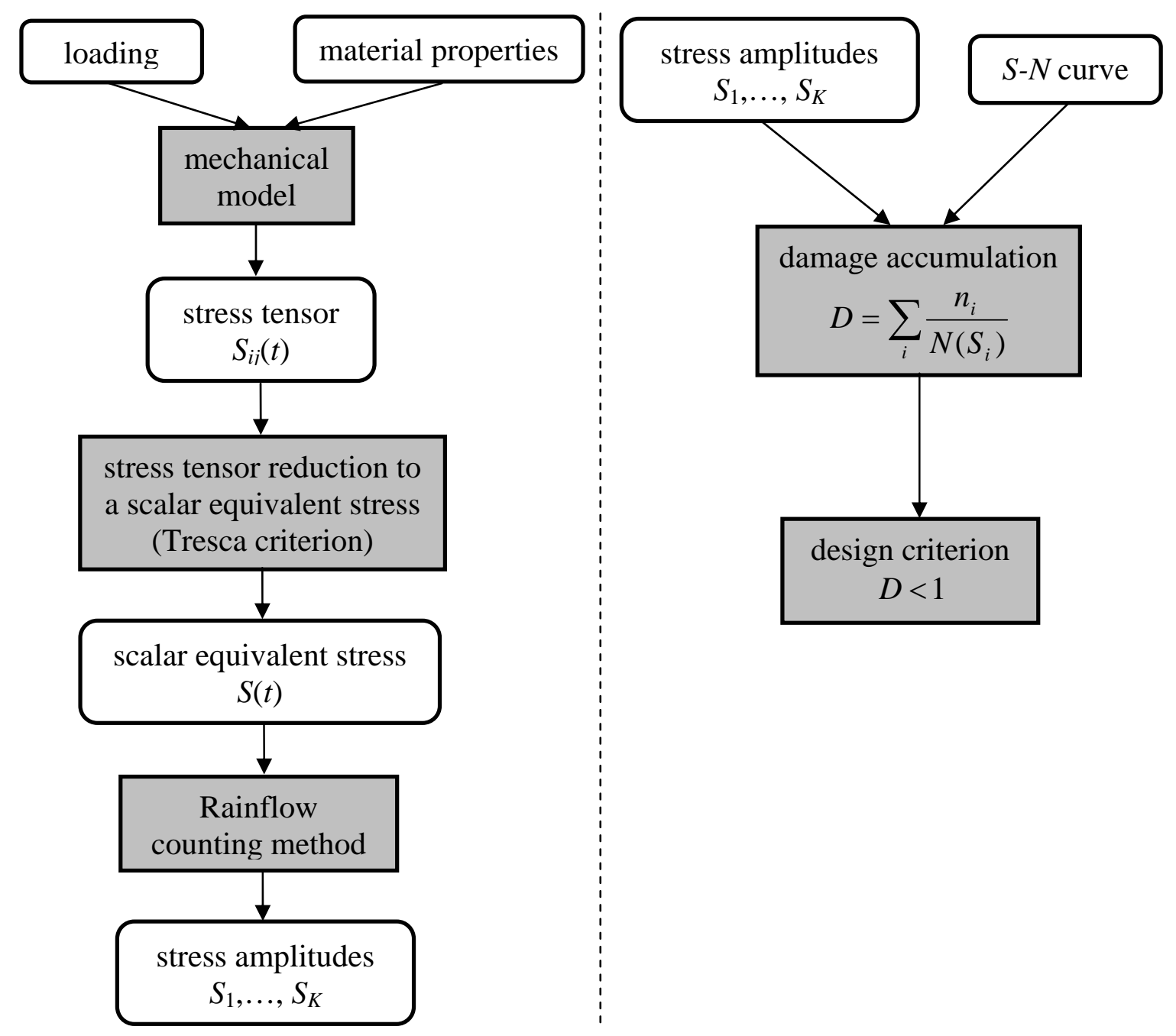

Fig. 1. Overview of the deterministic thermal fatigue design of nuclear components. 


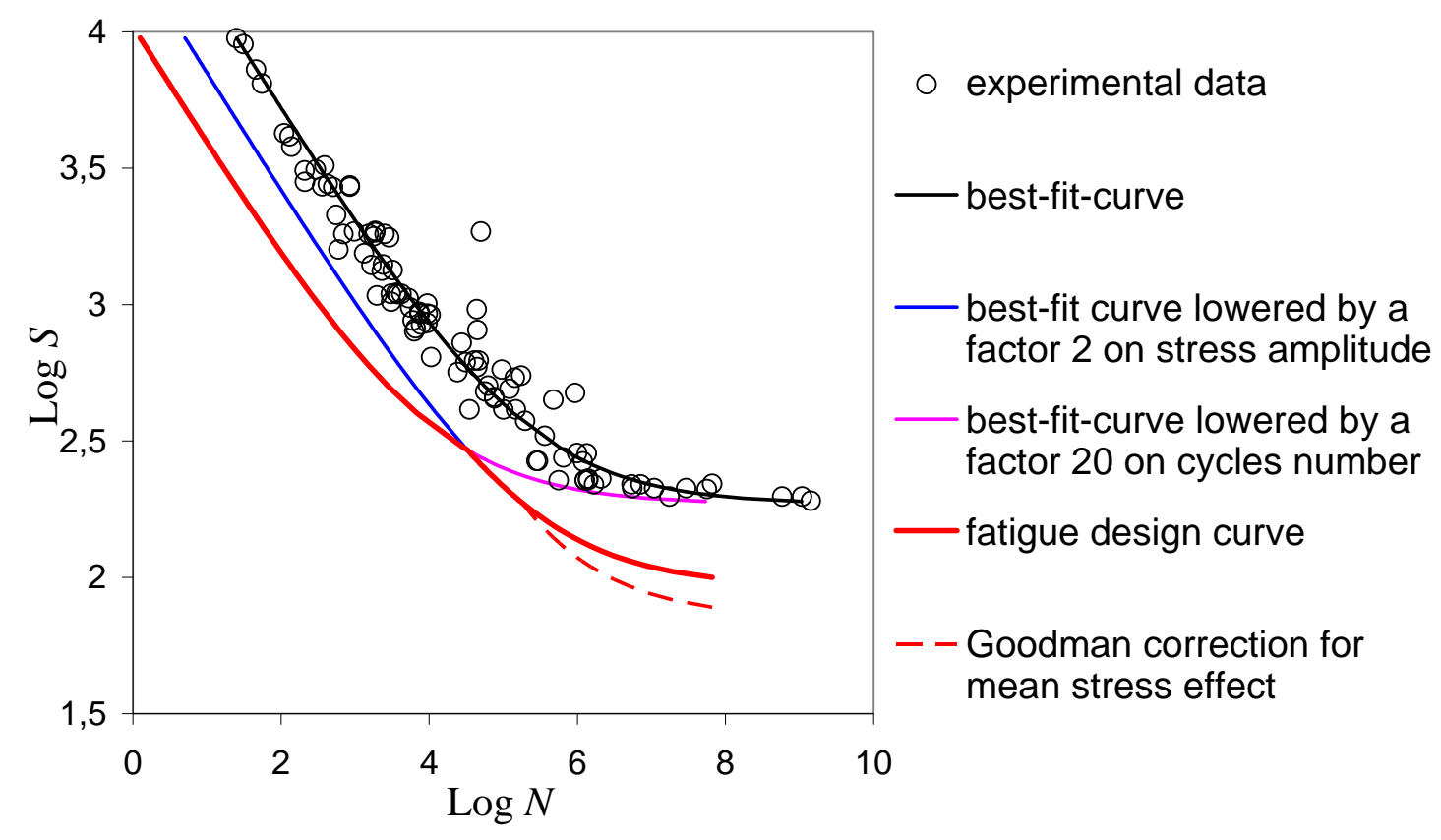

Fig. 2. Fatigue design curve and mean stress correction.

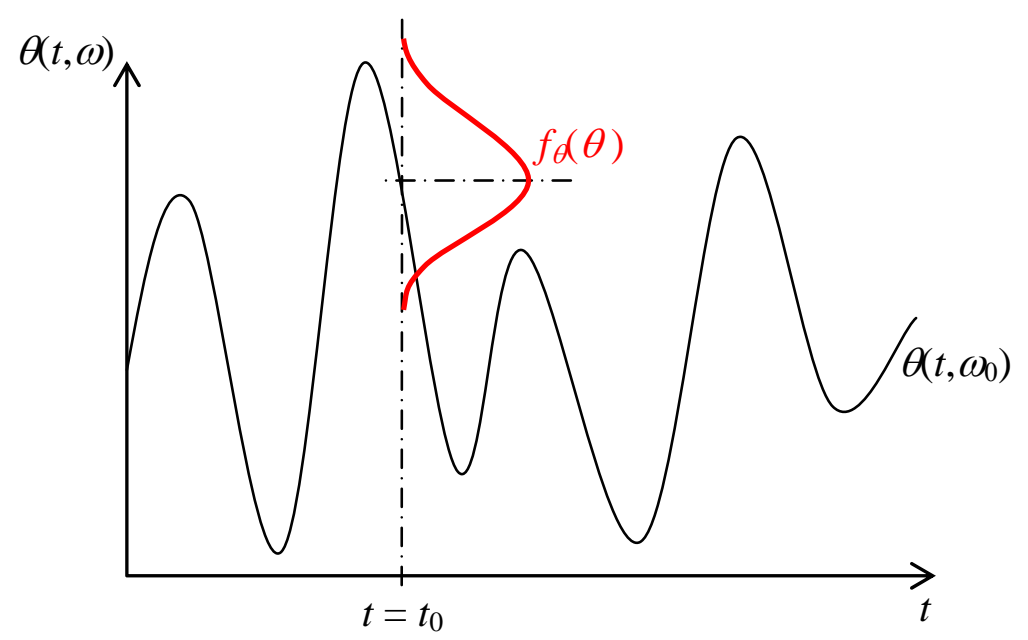

Fig. 3. Representation of a random process. 


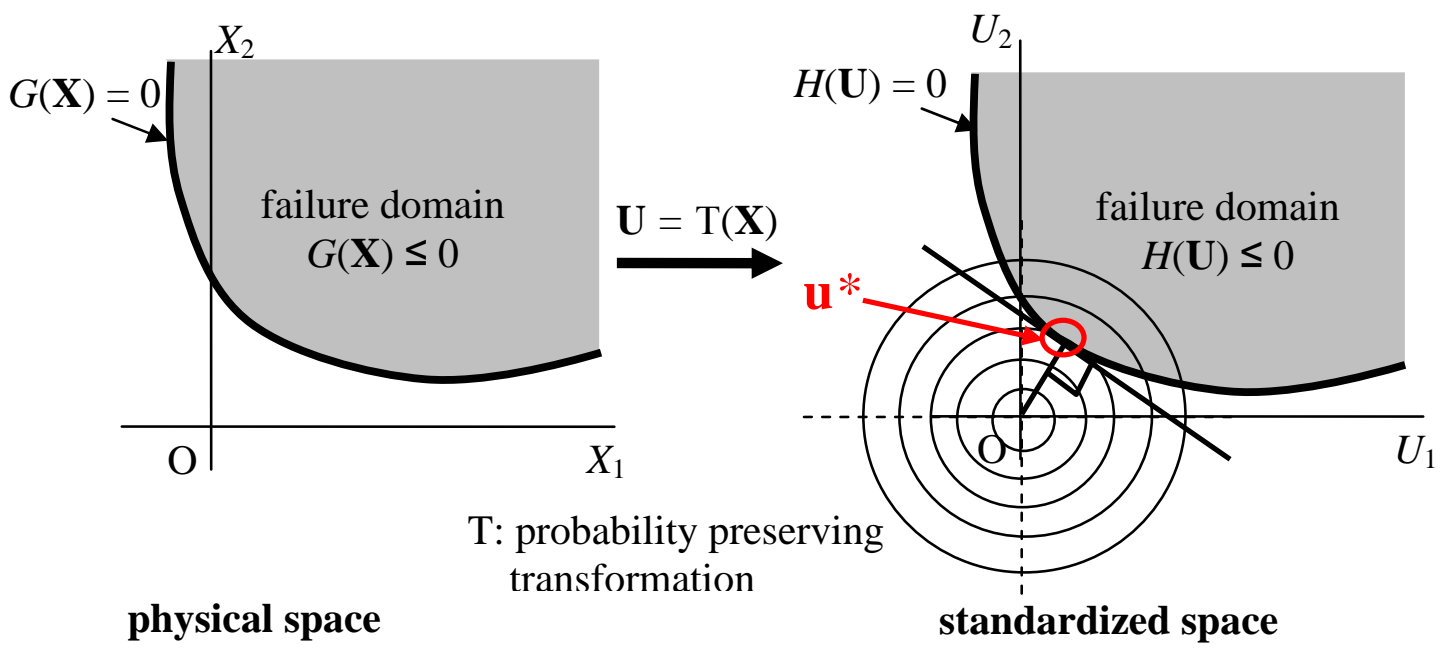

Fig. 4. Probability preserving transformation from the physical space to the standardized space. 
(a)

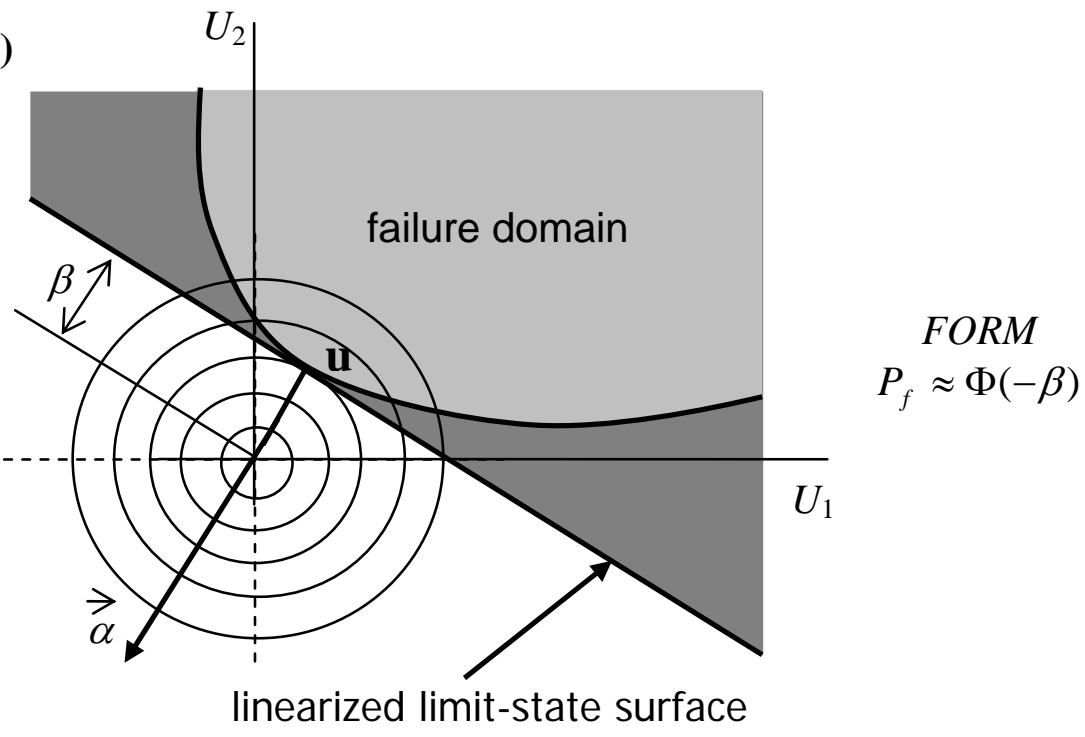

(b)

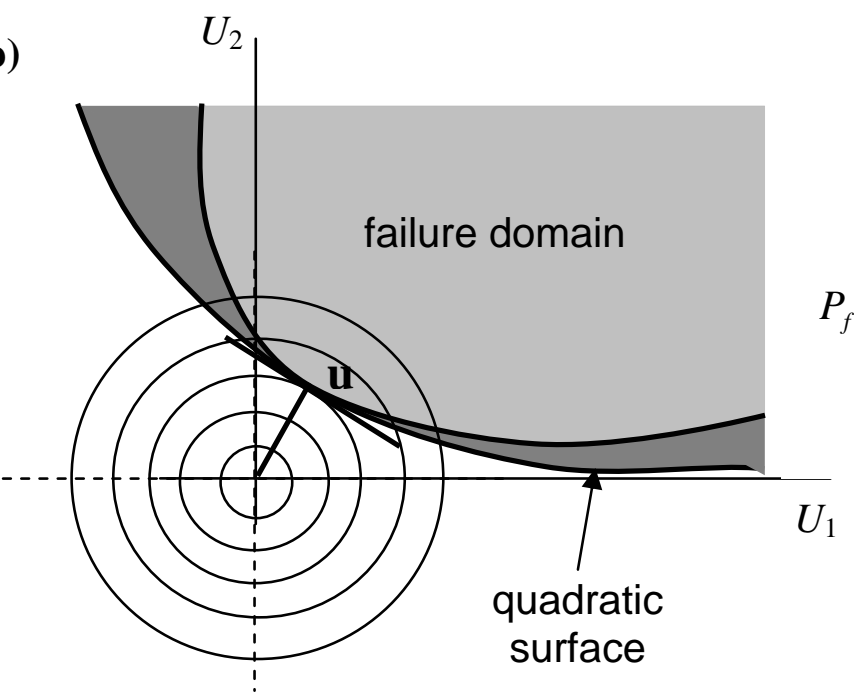

$$
P_{f} \approx \Phi(-\beta) \prod_{i=1}^{n-1}\left(1+\beta \kappa_{i}\right)^{-1 / 2}
$$

$\kappa_{i}$ : curvature at $\mathbf{u}^{*}$

$\kappa>0$ on the figure.

(c)

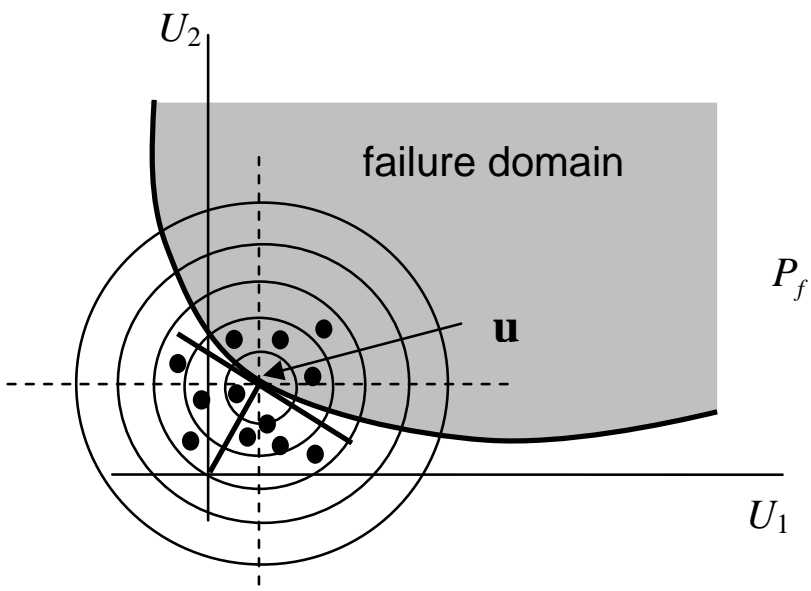

importance sampling $P_{f} \approx \frac{1}{K} \sum_{i=1}^{K} I[H(U) \leq 0] \frac{\varphi_{n}\left(u^{(i)}\right)}{\psi\left(u^{(i)}\right)}$ $\psi$. importance sampling distribution

Fig. 5. Failure probability approximation: (a) by FORM ; (b) by SORM ; (c) by importance sampling. 


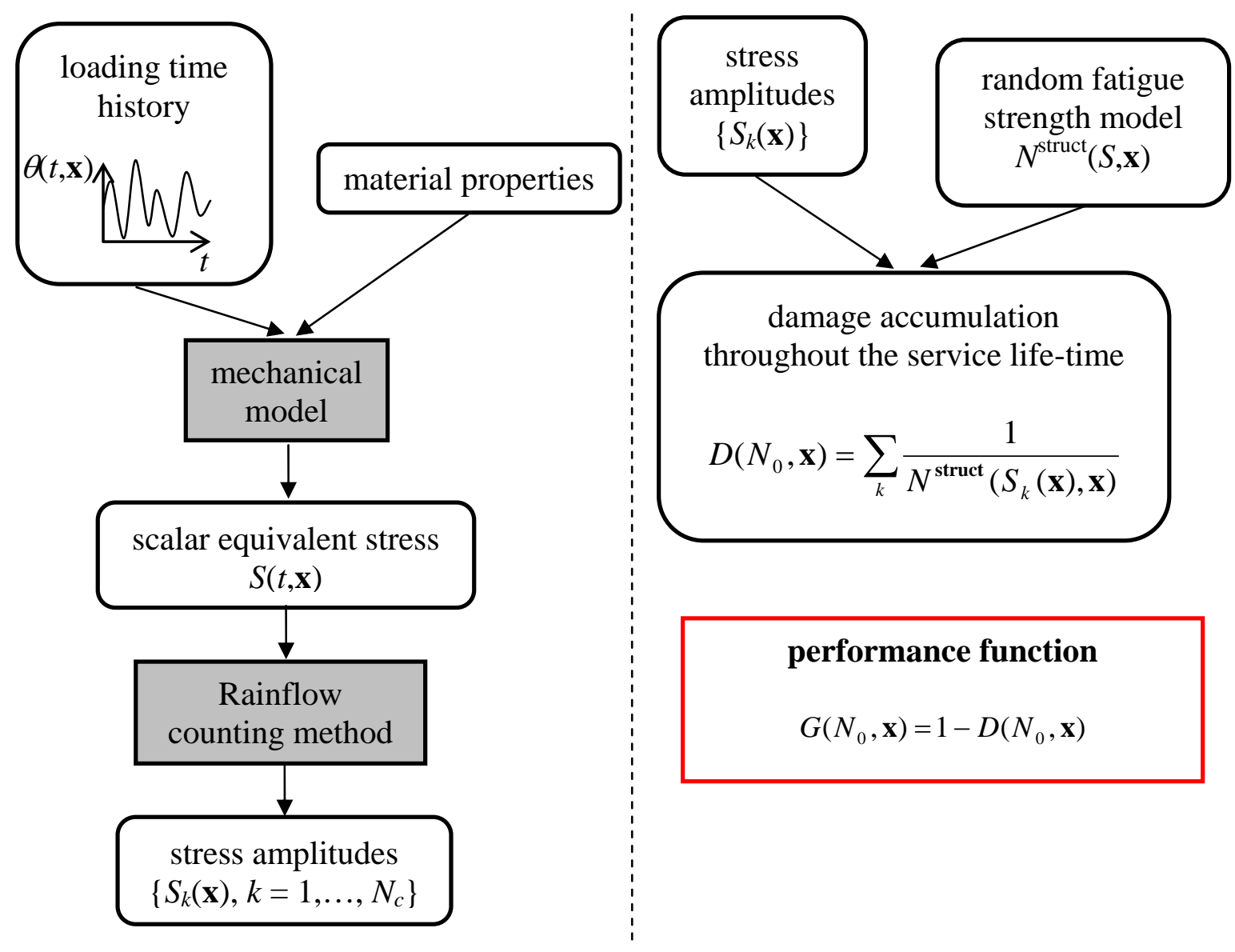

Fig. 6. Computational model for the reliability analysis in the time domain.

(a)

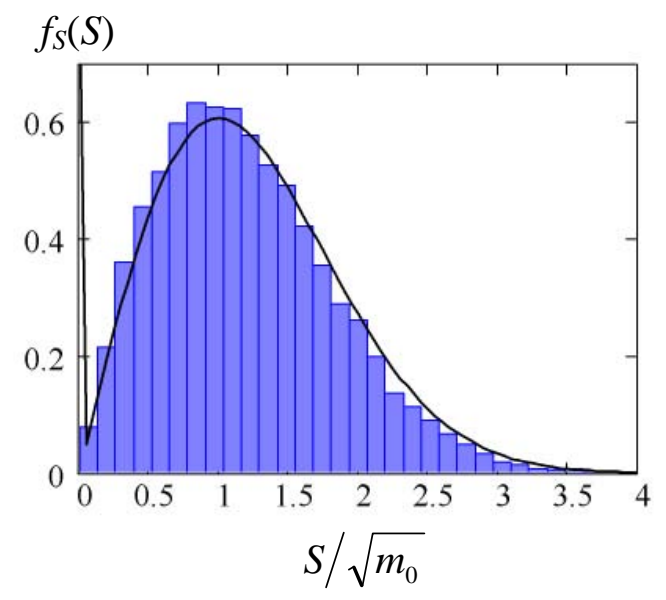

(b)

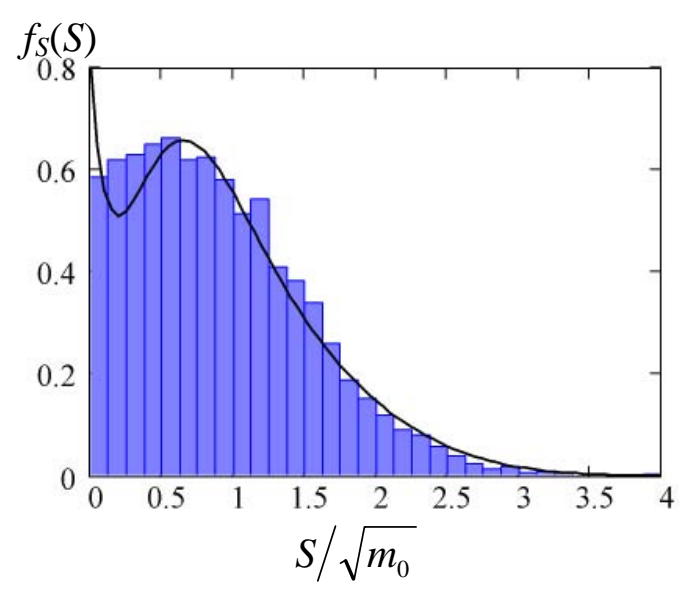

Fig. 7. Goodness of fit of the Dirlik formula to the Rainflow cycles for pseudo-white noise with (a) a narrow bandwidth and (b) a large bandwidth. 


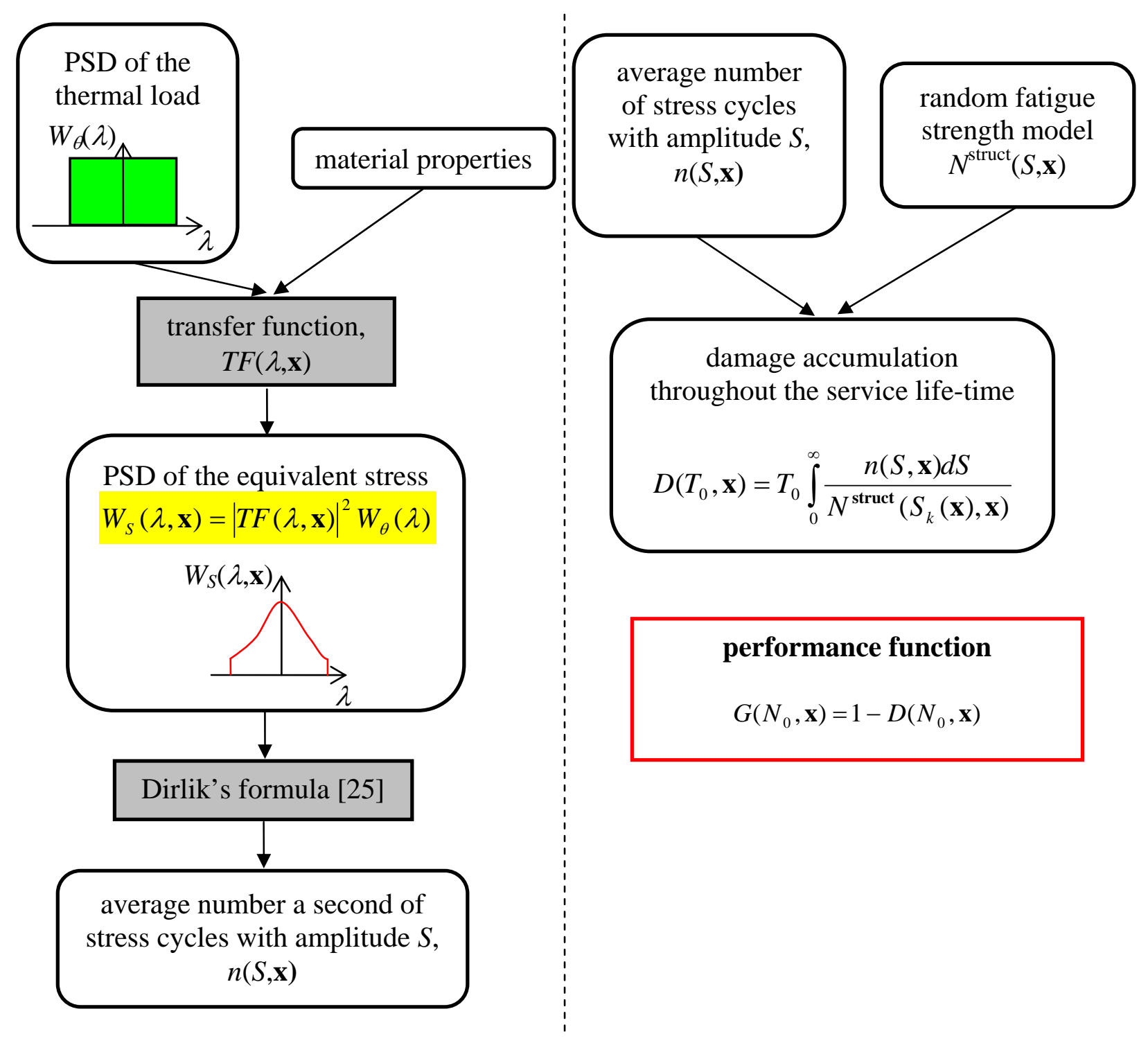

Fig. 8. Mechanical model for the reliability analysis in the frequency domain.
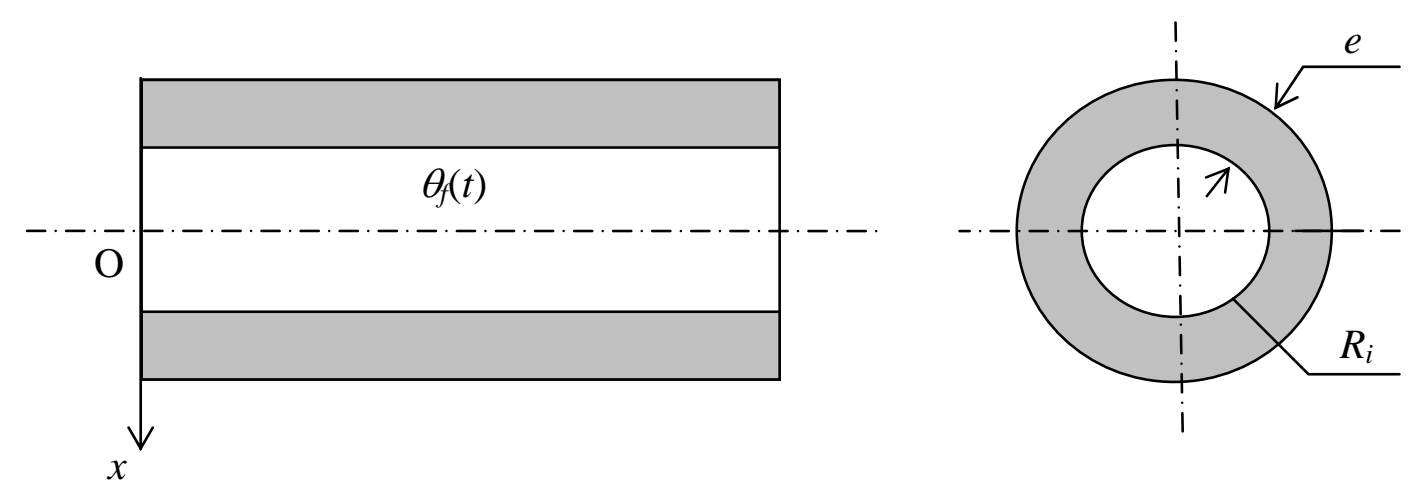

Fig. 9. Straight free end pipe. 

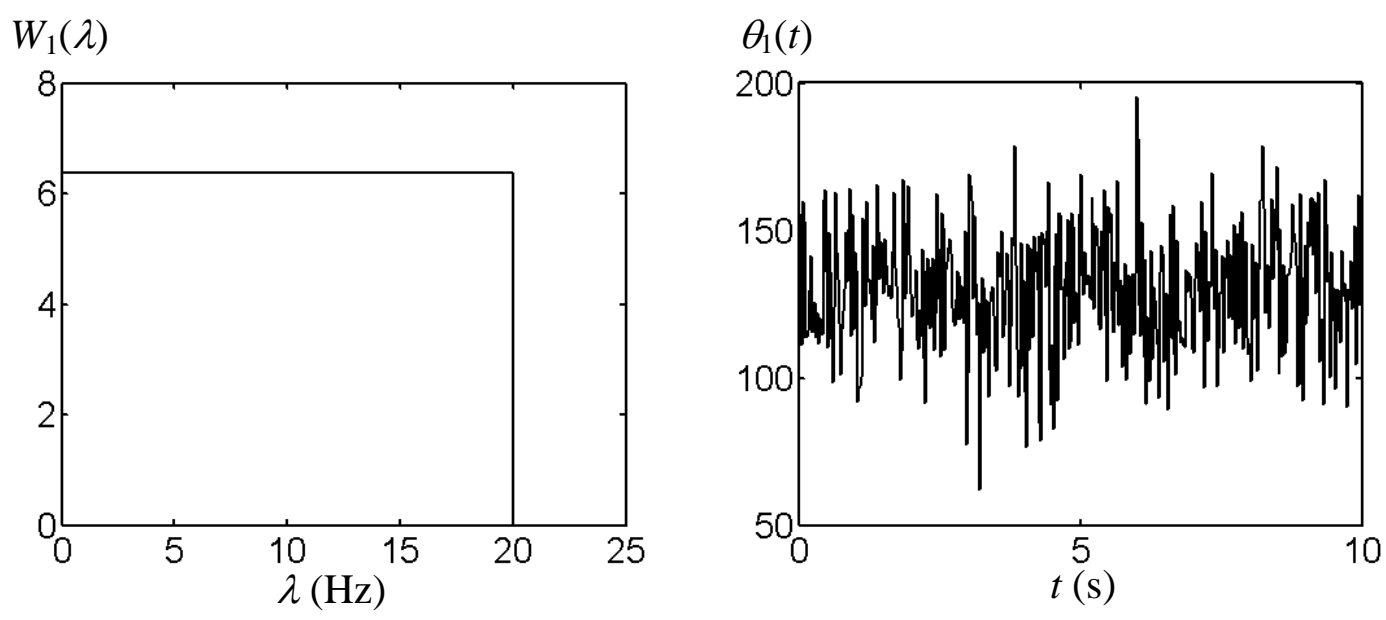

Fig. 10. Thermal loading model, $\theta_{1}(t, \omega)$.
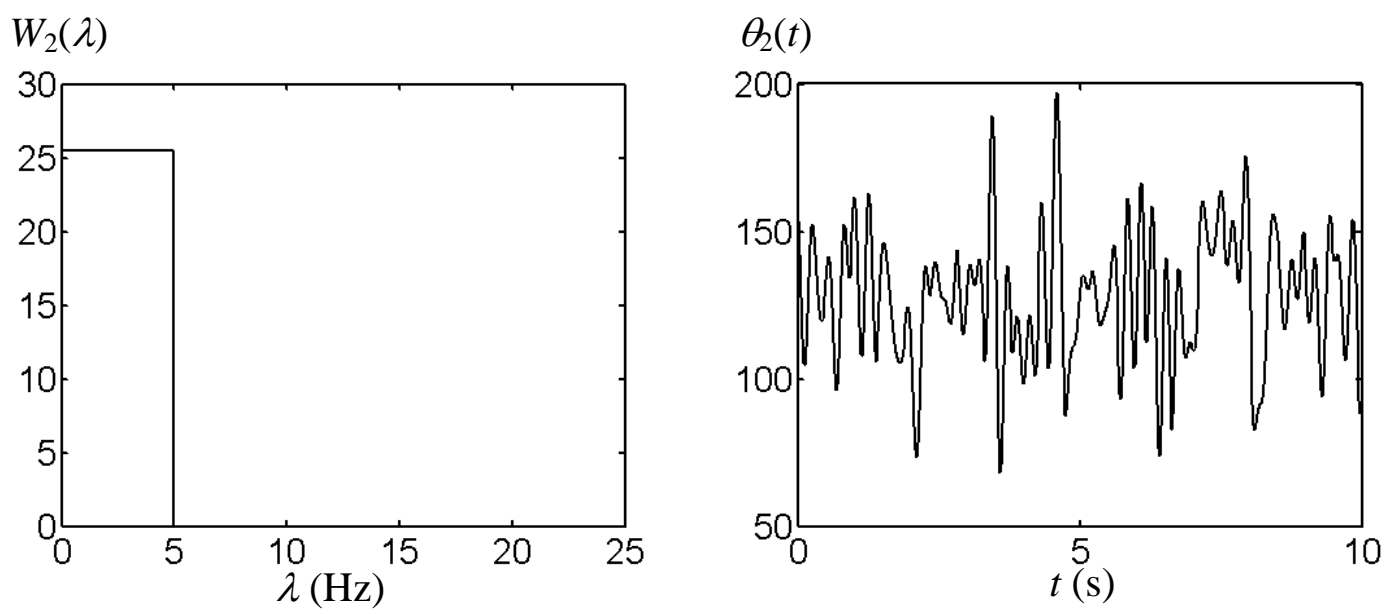

Fig. 11. Thermal loading model, $\theta_{2}(t, \omega)$. 


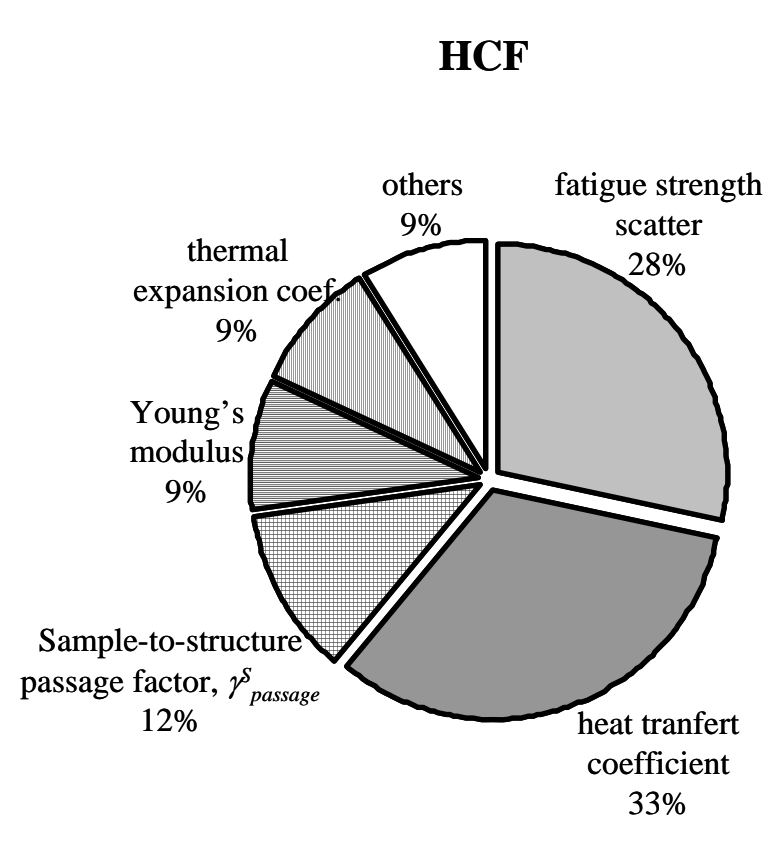

\section{LCF}

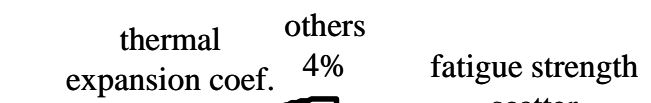

Fig. 12. Sensitivity factors for the thermal loading $\theta_{1}$ both in HCF and LCF regimes.

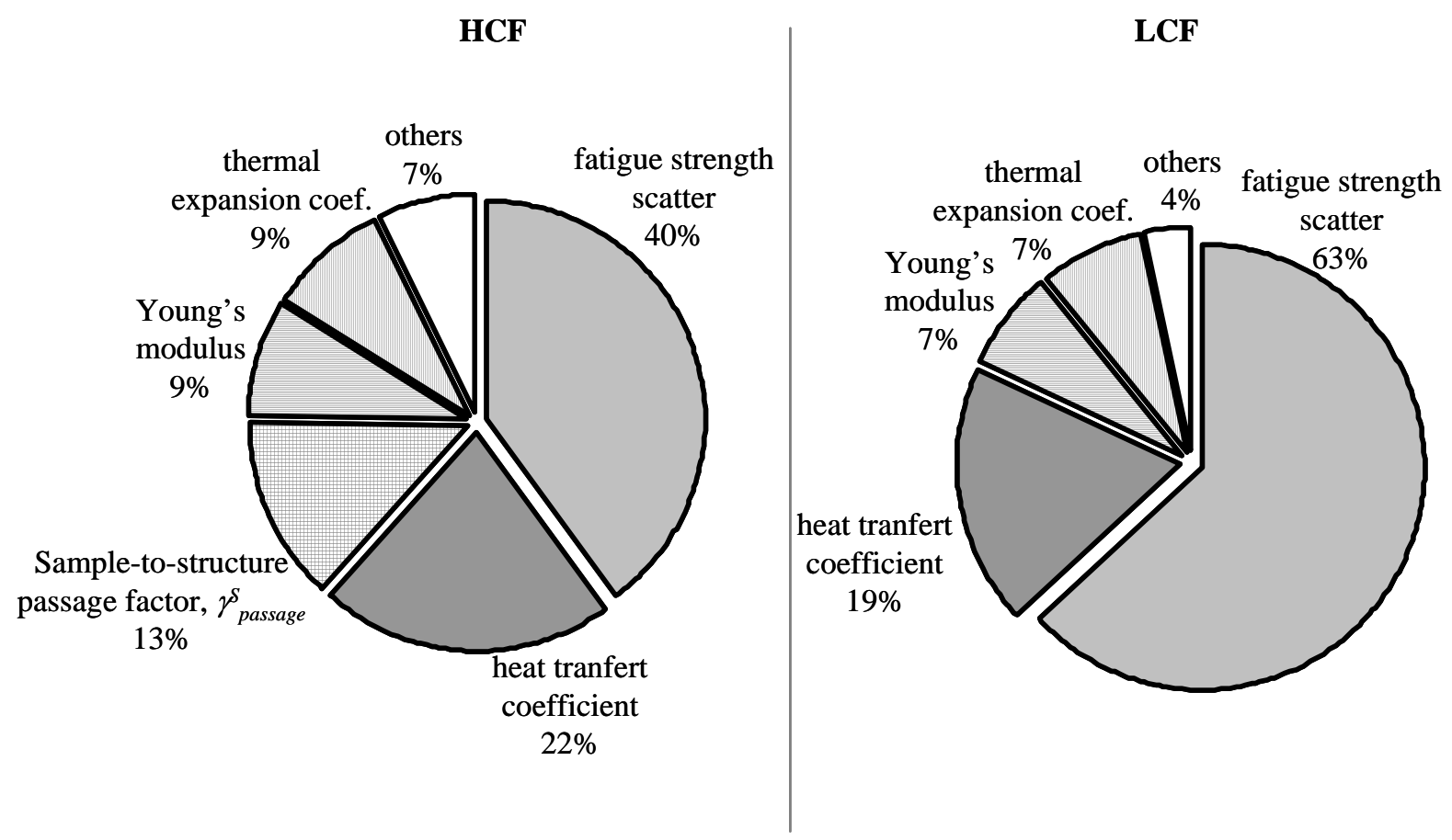

Fig. 13. Sensitivity factors for the thermal loading $\theta_{2}$ both in HCF and LCF regimes. 


\begin{tabular}{cccccc}
\hline$A$ & $B$ & $S_{D}(\mathrm{MPa})$ & $\delta$ & $D_{K}$ & $D_{K}{ }^{c}$ \\
\hline$-2,28$ & 24,06 & 185,80 & 0,09 & 0,11 & 0,14 \\
\hline
\end{tabular}

Table 1. Numerical results of the statistical treatment of fatigue test data.

\begin{tabular}{cccc}
\hline Variable name & Distribution & Mean & $\begin{array}{c}\text { Coef. of } \\
\text { variation }\end{array}$ \\
\hline Inner radius, $R_{i}$ & log-normal & $0.12827 \mathrm{~m}$ & $5 \%$ \\
Thickness, $e$ & log-normal & $9.2710^{-3} \mathrm{~m}$ & $5 \%$ \\
Young's modulus, $E$ & log-normal & $1.890810^{5} \mathrm{MPa}$ & $10 \%$ \\
Poisson ratio, $v$ & Bêta [0.2, 0.4] & 0.3 & $10 \%$ \\
Thermal expansion, $\alpha$ & log-normal & $1.69510^{-5}$ & $10 \%$ \\
Thermal conductivity, $L$ & log-normal & $16.645 \mathrm{~W} \cdot \mathrm{K}^{-1} \cdot \mathrm{m}^{-1}$ & $10 \%$ \\
Specific heat capacity, $\rho C_{p}$ & log-normal & $4.02410^{6} \mathrm{~W}^{-1} \mathrm{~K}^{-1} \cdot \mathrm{m}^{-3}$ & $10 \%$ \\
Heat transfer coef., $h$ & log-normal & $20,000 \mathrm{~W} \cdot \mathrm{K}^{-1} \cdot \mathrm{m}^{-2}$ & $30 \%$ \\
Yield strength, $S_{y}$ & log-normal & $188 \mathrm{MPa}$ & $10 \%$ \\
Ultimate tensile strength, $S_{u}$ & $\log$-normal & $496 \mathrm{MPa}$ & $10 \%$ \\
$\gamma_{\text {passage }}^{S}$ & Bêta [1,2] & 1.68 & $10 \%$ \\
$\gamma_{\text {passage }}^{N}$ & Bêta [7,11] & 9.39 & $10 \%$ \\
Fatigue strength scatter, $\xi$ & normal & 0 & std. dev. $=1$ \\
\hline
\end{tabular}

Table 2. Statistical properties of the input random variables.

\begin{tabular}{c|c|cc|cc}
\hline \multirow{2}{*}{ Reliability analysis } & \multicolumn{2}{|c|}{$\theta_{1}(t, \omega)$} & \multicolumn{2}{c}{$\theta_{2}(t, \omega)$} \\
\cline { 2 - 6 } & & HCF & LCF & HCF & LCF \\
\hline \multirow{3}{*}{ Time domain } & FORM & 2.28 & 2.48 & 1.36 & 1.99 \\
\cline { 2 - 6 } & SORM & 2.35 & 2.50 & 1.44 & 2.01 \\
\cline { 2 - 6 } & $I S$ & 2.36 & 2.48 & 1.43 & 2.02 \\
\hline \multirow{3}{*}{ Frequency domain } & FORM & 1.88 & 2.07 & 1.35 & 1.99 \\
\cline { 2 - 6 } & SORM & 1.95 & 2.07 & 1.42 & 2.01 \\
\cline { 2 - 6 } & IS & 1.99 & 2.07 & 1.41 & 2.01 \\
\hline
\end{tabular}

Table 3. Reliability indices obtained by FORM, SORM and importance sampling (IS) methods. 